\title{
An apoplastic peptide signal activates salicylic acid signalling in maize
}

Sebastian Ziemann ${ }^{1}$, Karina van der Linde ${ }^{2 \dagger}$, Urs Lahrmann ${ }^{3}$, Beyda Acar ${ }^{1}$, Farnusch Kaschani ${ }^{4}$, Tom Colby ${ }^{5}$, Markus Kaiser ${ }^{4}$, Yezhang Ding ${ }^{6}$, Eric Schmelz ${ }^{6}$, Alisa Huffaker ${ }^{6}$ Nicholas Holton ${ }^{7}$, Cyril Zipfel ${ }^{7}$ and Gunther Doehlemann ${ }^{1 *}$

1Botanical Institute and Cluster of Excellence on Plant Sciences (CEPLAS), University of Cologne, BioCenter, Zuelpicher Str. 47a, 50674 Cologne, Germany.

2Department of Biology, Stanford University, 385 Serra Mall, Stanford, CA 94305-5020, USA.

${ }^{3}$ Fraunhofer Institute for Toxicology and Experimental Medicine ITEM, Division of Personalized Tumor Therapy, 93053 Regensburg, Germany.

${ }^{4}$ Centre for Medical Biotechnology, Chemical Biology, Faculty of Biology, University Duisburg-Essen, 45117 Essen, Germany.

Max Planck Institute for Biology of Ageing, Joseph-Stelzmann-Str. 9b, 50931 Cologne, Germany.

'Section of Cell and Developmental Biology, University of California at San Diego, La Jolla, CA 92093.

${ }^{7}$ The Sainsbury Laboratory, Norwich Research Park, Norwich, NR4 7UH, UK. ${ }^{\dagger}$ Current address: Cell Biology and Plant Biochemistry, University of Regensburg, Universitätsstraße 31, 93053 Regensburg, Germany

*correspondence to: Gunther Doehlemann; email: g.doehlemann@uni-koeln.de

Running title: Plant immune signalling peptide

The author responsible for distribution of materials integral to the findings presented in this article is: Gunther Doehlemann (g.doehlemann@uni-koeln.de) 


\section{Abstract}

Control of plant pathogen resistance or susceptibility largely depends on the promotion of either

cell survival or cell death. In this context, papain-like cysteine proteases (PLCPs) regulate plant defence to drive cell death and protection against biotrophic pathogens. In maize (Zea mays), PLCPs are crucial in the orchestration of salicylic acid (SA)-dependent defence signalling. Despite this central role in immunity, it remains unknown how PLCPs are activated, and which downstream signals they induce to trigger plant immunity. Here, we present the discovery of an immune signalling peptide, Zea mays ịmmune signalling peptide 1 (Zip1). A mass spectrometry approach identified the Zip1 peptide being produced after salicylic acid (SA) treatment. In vitro studies using recombinant proteins demonstrate that PLCPs are required to release bioactive Zip1 from its propeptide precursor (PROZIP1). Strikingly, Zip1 treatment strongly elicits SA accumulation in maize leaves. Moreover, RNAseq based transcriptome analyses revealed that Zip1 and SA treatments induce highly overlapping transcriptional changes. Consequently, Zip1 promotes the infection of the necrotrophic pathogen Botrytis cinerea in maize, while it reduces virulence of the biotrophic fungus Ustilago maydis. Together, Zip1 represents the previously missing signal that is released by PLCPs to activate SA defence signalling. 


\section{Introduction}

3 Plants face a wide range of biotic threats including viruses, bacteria, insects and fungi.

4 Protective processes including local and systemic defences are mediated in part by plant

5 proteases that additionally regulate stomatal development, embryogenesis, and cuticle

6 deposition ${ }^{1}$. Importantly, proteases from diverse catalytic classes have been associated with

7 immunity in plants ${ }^{1}$. The apoplastic aspartic protease CDR1 (Constitutive Disease

8 Resistance1), for instance, induces local and systemic defence responses in Arabidopsis

9 thaliana. Increased bacterial susceptibility to Pseudomonas syringae occurs in cdr1 mutants

10 whereas $C D R 1$ overexpression results in enhanced resistance ${ }^{2}$. Another example of proteases

11 involved in plant immunity is the tomato subtilisin-like protease P69 ${ }^{3}$. Out of six characterized

12 isoforms, two (P69B and P69C) are transcriptionally upregulated by the defence hormone

13 salicylic acid (SA) and by infection with $P$. syringae, suggesting that serine proteases are

14 important during pathogenesis ${ }^{4}$. In addition, the $A$. thaliana serine protease SITE-1 PROTEASE

15 (S1P) cleaves RAPID ALKALIZATION FACTOR23 (RALF23) to inhibit plant immunity via the 16 malectin-like receptor kinase FERONIA (FER) ${ }^{5}$.

17 Among the classes of plant proteases, the papain-like cysteine proteases (PLCPs) are central

18 hubs in the regulation of programmed cell death and plant immunity ${ }^{1,6}$. A crucial role of PLCPs

19 in plant immunity is highlighted by the discovery that evolutionary unrelated plant pathogens

20 have independently evolved effector proteins that target PLCPs to promote virulence. For

21 instance, the tomato PLCP RCR3 (Required for Cf-2-Dependent Disease Resistance3) is

22 targeted by the Avr2 (Arvirulence-2) effector protein of the fungal pathogen Passalora fulva

23 (previously Cladosporium fulvum) ${ }^{7}$. In addition, it is inhibited by the cystatin-like effectors EPIC1

24 (Extracellular Cystatin-like Protease Inhibitor1) and EPIC2B of the oomycete pathogen

25 Phytopthtora infestans and the allergen-like effector Gr-VAP1 (Venom Allergen-like effector 26 Protein1) of the nematode Globodera rostochiensis ${ }^{8,9}$. 
27 Apoplastic PLCPs have significant roles in the activation of diverse plant defence responses.

28 Further, the regulation of plant immunity also commonly involves the fine-tuned interplay of 29 phytohormones such as salicylic acid (SA), jasmonic acid (JA) and ethylene (ET). Among 30 defence-related phytohormones, SA is a key player that orchestrates responses to both biotic 31 and abiotic stresses ${ }^{10,11}$ and extensive studies have detailed the role of SA in innate immune 32 signalling ${ }^{12}$. In general, research in $A$. thaliana and Nicotiana benthamiana has revealed that 33 SA signalling promotes efficient defence activation against biotrophic pathogens, whereas 34 necrotrophic pathogens are sensitive to JA/ET-dependent defence signalling. Early publications 35 emphasized the potential for SA-mediated antagonism for the strong inhibition of wound36 induced JA signalling ${ }^{13,14}$. Beyond classical phytohormones, endogenous plant peptides can act 37 on different levels of signal amplification relevant to JA/ET dependent defence signalling ${ }^{10,15}$. In A. thaliana and maize, small peptides can be released from larger pro-peptides to act as damage-associated molecular patterns (DAMPs) ${ }^{16-18}$. In maize, transcripts encoding the PLANT ELICITOR PEPTIDE 1 (ZmPEP1) precursor protein (ZmPROPEP1) display induced expression

41 following JA treatment ${ }^{16}$. In $A$. thaliana, AtPEP1 activates pathogen defence responses and 42 confers disease resistance when ectopically expressed ${ }^{18}$. Likewise in maize, ZmPEP1 43 promotes the production of JA, ET, and defence gene expression. Consequently, pretreatment 44 of maize with ZmPEP1 leads to enhanced resistance to necrotrophic fungal pathogens. Thus, 45 PEPs from $A$. thaliana and maize are functionally conserved DAMPs regulating JA-associated 46 innate immune responses in diverse plant species ${ }^{16,17}$.

47 The maize pathogen Ustilago maydis is a biotrophic fungus, which induces formation of tumors 48 on all aerial parts of its host plant ${ }^{19}$. At the onset of infection, $U$. maydis transiently induces 49 pathogen-associated molecular pattern (PAMP)-triggered immunity (PTI) responses, including $50 \quad$ PR-gene expression. In the compatible interaction with maize, these responses are suppressed 51 upon fungal penetration and accommodation of biotrophic infection structures 24 hours after 52 infection ${ }^{20}$. In incompatible interactions, $U$. maydis induces typical plant immune responses 
53 including the rapid accumulation of reactive oxygen species (ROS), induction of $P R$-gene 54 expression, SA-associated defence responses and programmed cell death ${ }^{20-22}$. Successful $U$. maydis infection depends on the induction of the maize cystatin CC9, which inhibits a set of SAinduced, apoplastic PLCPs ${ }^{23}$. In turn, activity of these apoplastic enzymes can trigger the activation of SA-associated defence signalling ${ }^{23}$. Three maize PLCPs (CP1, CP2 and XCP2) are also inhibited by the $U$. maydis effector Pit2, and the inhibitory activity of this protein is essential for virulence of the pathogen ${ }^{24}$. While these findings demonstrate the important role of apoplastic PLCPs for the regulation of plant immunity, key questions remain unanswered. For example, how do apoplastic PLCPs induce downstream SA signalling? What are the targets of PLCPs? Are signals released by PLCPs? What downstream signalling pathways are involved? Based on previous findings, we hypothesized that the activation of SA-related defences by PLCPs is mediated by the release of apoplastic peptides that in turn act as signals to activate downstream responses. In the present study we describe the identification and functional characterization of a novel peptide which is released by PLCP-activity and induces SA accumulation and signalling in maize.

\section{Results}

\section{Peptides present in SA-treated apoplastic fluid induce defence responses}

To examine if bioactive maize peptides are released by the activity of PLCPs, leaves were treated with SA to first promote apoplastic protease activity. Confirming previous results ${ }^{23}$, apoplastic fluid of SA-infiltrated leaves showed strongly induced PLCP activity compared to mock samples $24 \mathrm{~h}$ after treatment (Fig. S1). Apoplastic fluids of both SA- and mock-treated leaves were subjected to Amicon ${ }^{\circledR}$ filtration to separate small peptides $(<10 \mathrm{kDa})$ from proteins. Peptide fractions of SA-treated and mock treated leaves were then re-introduced into naïve plants by leaf infiltration to test for activity. After infiltration, transcriptional changes of SA-related PR-genes were analysed by qRT-PCR at $24 \mathrm{~h}$ (Fig. 1A). Peptide fractions from SA-treated 
leaves resulted in a significant induction of the previously identified maize SA marker genes ZmPR3, ZmPR4 and ZmPR5. In contrast to SA-related markers, transcript levels of JA-induced $Z m C C 9^{23}$ were not affected by apoplastic peptides (Fig $1 \mathrm{~A}$ ). This result suggests that activity of SA-induced PLCPs can release peptide(s) into the apoplastic fluid, which in turn activate SA mediated processes.

\section{Identification of Zea mays immune signalling peptide 1 (Zip1)}

To identify bioactive peptide candidates, fractions $(<10 \mathrm{kDa})$ from apoplastic fluids of SA- and mock treated plants were analysed by liquid chromatography mass spectrometry (LC-MS) (Fig. S2A). MS-identified, SA-induced peptides were synthesized and infiltrated into naïve maize leaves to test their ability to induce $P R$-gene expression in vivo $24 \mathrm{~h}$ after infiltration. In parallel, plants were treated with $2 \mathrm{mM} \mathrm{SA}$ as a positive control (Fig. S2B, S3). qRT-PCR was done for the SA markers ZmPR3, ZmPR4, ZmPR5, as well as ZmPRm6b, and ZmPR10 ${ }^{23,25,26}$. Out of four candidates, this assay identified one peptide eliciting the accumulation of $P R$-gene transcripts to a similar level compared to SA (Fig. 1B). This 17 amino acid peptide ['EGESELKLATQGASVRR] was termed Zea mays inmune signalling peptide 1 (Zip1). To test whether Zip1 induced PR-gene expression is sequence specific, a mutated peptide version (Zip1 mut) was generated, in which the N-terminal charged amino acids Glu and Lys were substituted to neutral Ala (Fig. 1B). In the maize leaf assay for elicited $P R$-gene expression, the Zip1 $1_{\text {mut }}$ peptide is completely inactive (Fig. 1B), indicating that the charged $\mathrm{N}$-terminus is required for the induction of Zip1-induced defence signalling. In contrast to the Zip1 mut peptide. a native Zip1 version with a three amino acid $\mathrm{N}$-terminal extension (QPW) triggered PR-gene induction similar to the 17aa version (Fig S3), indicating potential variability for the $\mathrm{N}$-terminal boundary of Zip1. 


\section{Zip1 is released from a pro-peptide by PLCP activity}

106 A MASCOT algorithm-based maize genome search for Zip1 identified an annotated open 107 reading frame for a precursor protein (AC210027.3_FGP003) that was named PROZIP1. . The

108137 aa protein is predicted for unconventional secretion (SecretomeP 2.0; 109 http://www.cbs.dtu.dk/services/SecretomeP/) but does not contain any known domains (Expasy 110 PROSITE, https://prosite.expasy.org/). A qRT-PCR experiment showed that transcript levels for 111 PROZIP1 are neither induced by Zip1, nor by SA, (Fig. S2C) which indicates a post112 transcriptional regulation of its activity. To test if Zip1 can be released from PROZIP1 by maize 113 PLCPs, PROZIP1 was cloned and fused to an N-terminal HA-tag for heterologous production in 114 Escherichia coli (Fig. S4) and co-incubated with apoplastic fluid from SA treated maize plants. 115 Co-incubation resulted in a time-dependent cleavage of PROZIP1, which can be blocked by the 116 addition of E-64 ${ }^{27}$, a specific PLCP inhibitor (Fig 2A). This result indicates that PROZIP1 is a 117 substrate of SA-activated maize PLCPs. To test, if individual maize proteases are capable of PROZIP1 cleavage, co-incubation assays with the previously identified ${ }^{23}$ apoplastic maize PLCPs CP1, CP2, CatB and XCP2 were performed. PLCPs were heterologously expressed in $N$. benthamiana and protease activity was normalized and monitored via activity based protein 121 profiling (ABPP) ${ }^{28}$ using the fluorescent PLCP-specific probe MV-202 ${ }^{29}$ (Fig. 2B: chemical 122 structure Fig. S1A). Co-incubation of equal amounts of active individual PLCPs resulted in 123 cleavage of PROZIP1 by CP1 and CP2, but not by CatB and XCP2 (Fig. 2C). This result shows 124 that the maize PLCPs CP1 and CP2 are required for processing of PROZIP1.

125 PROZIP1 contains six RR/FR motifs that are predicted to be potential protease cleavage sites 126 due to their hydrophobic and dibasic properties (Fig 2D) ${ }^{30,31}$. Maize PLCP activity towards these

127 sides was tested with different fluorescent substrates that identified Arg-Arg and Phe-Arg 128 sequence motifs as most efficient cleaved sites (Fig. S5). To test if cleavage at these predicted 129 sites actually releases Zip1, two different PROZIP1 versions with substituted RR/FR motifs were 130 generated and purified from E. coli (Fig. 2D and S4). In PROZIP1Mut ${ }^{\mathrm{CS}}$ all six di-arginine motifs 
131 were substituted into di-alanines. A second version of the propeptide (PROZIP1Mut ${ }^{\text {CS2}}$ )

132 contained only mutations of the two predicted cleavage sites surrounding the Zip1 peptide (Fig.

133 2D), while the remaining four sites remained unaffected. Apoplastic fluid containing active

134 PLCPs, as well as individual proteases expressed in $N$. benthamiana were co-incubated with

135 PROZIP1mut ${ }^{\mathrm{CS} / \mathrm{CS} 2}$. Unlike the native propeptide, PROZIP1mut ${ }^{\mathrm{CS}}$ was not processed upon PLCP

136 treatment, which indicates that the mutated sites are required for PLCP-induced cleavage. For

137 PROZIP1mut ${ }^{\mathrm{CS} 2}$, the $\mathrm{\alpha}-\mathrm{HA}$ immunoblot showed PLCP-dependent processing (Fig. 2C, Fig.

138 S5B), reflecting that this mutant version carries four of the six predicted cleavage sites.

139 To test if the in vitro processed PROZIP1 releases biologically active forms of Zip1, a large140 scale cleavage assay with subsequent extraction of peptides of a molecular weight $<10 \mathrm{kDa}$ was 141 performed. Naïve plants were infiltrated with these peptide fractions of PROZIP1 treated with 142 active proteases or E-64-inhibited proteases as negative control. Subsequent qRT-PCR 143 revealed a significant upregulation of $P R$-genes triggered by PROZIP1 peptide fractions that 144 were incubated with PLCPs (Fig 3A). This induction of PR-genes was not observed when 145 PLCPs were inhibited with E-64 prior to co-incubation with PROZIP1, demonstrating a PLCP146 dependent release of active Zip1 (Fig 3A). In addition, co-incubation of both PROZIP1mut ${ }^{\mathrm{CS}}$ and 147 PROZIP1mut ${ }^{\mathrm{CS} 2}$ with active PLCPs did not result in release of peptides inducing significant $P R$ 148 gene expression. This confirms that i) the RR/FR motifs in PROZIP1 are crucial for the release 149 of the signalling peptide Zip1, ii) PROZIP1 contains no additional PR-gene activating peptides 150 besides Zip1, and iii) the activity observed is most likely not caused by small residual amounts 151 of SA itself (Fig 3A).

\section{Zip1 activates maize PLCPs}

154 To further characterize downstream responses triggered by Zip1, we tested the rapid production 155 of reactive oxygen species (ROS), a typical immune response induced upon perception of 156 PAMPs or damage-associated molecular patterns (DAMPs), such as elf18, flg22, chitin or 
AtPEP1 ${ }^{32-35}$. For this, maize leaf discs were treated with $5 \mu \mathrm{M}$ Zip1. While both $1 \mu \mathrm{M}$ chitin and $1 \mu \mathrm{M}$ flg22 elicited typical PAMP-induced ROS bursts, Zip1 treatment did not cause detectable production of ROS (Fig. S6). Next, phosphorylation of maize MAP-kinases was tested by western blotting. However, in contrast to chitin and flg22, Zip1 did not cause any phosphorylation detectable with an a-Phospho p44/p42 antibody (Fig. S6B). Thus, in the

162 context of rapid ROS production and MAPK phosphorylation, Zip1 lacks common overlapping 163 PTI responses in maize.

164 We previously demonstrated the reciprocal activation of PLCPs and SA signalling in maize ${ }^{23}$. 165 To explore the potential direct influence of Zip1 on PLCPs, ABPP assays were performed on 166 apoplastic extracts from maize leaves $24 \mathrm{~h}$ after treatment with SA, Zip1 or Zip1 mut, respectively.

167 While an ABPP of ZIP1 $1_{\text {mut }}$-treated samples showed only weak PLCP activity compared to mock 168 samples, Zip1 treated leaves displayed strong induction of apoplastic PLCP activity, which is similar to samples that were infiltrated with SA (Fig. 3B). A possible explanation for this result could be an exosite activation of PLCPs by direct interaction with the Zip1 peptide ${ }^{36}$. To test if

171 PLCPs are directly activated by the Zip1 peptide, leaf extracts of SA- and mock- treated leaves 172 were incubated with Zip1 and subsequently labelled with DCG-04. Co-incubation with Zip1 in 173 vitro did not result in elevated DCG-04 labelling (Fig. 3B) which suggests an indirect Zip1174 mediated PLCP activation via a so far unknown signalling cascade. Our results point towards a 175 positive feedback loop in which Zip1 is released from PROZIP1 by SA-activated PLCPs and, in 176 turn, induces the activity of these proteases.

\section{Zip1 is a functional elicitor of SA signalling}

179 Zip1 is an endogenous maize peptide that induces transcriptional activation of SA marker 180 genes. This finding raises the question, whether Zip1 ultimately has a direct influence on SA 181 levels in maize. To this end, SA contents were determined by LC/MS/MS measurements of 182 maize leaves treated with Zip1. Mock-treated tissue, as well as Zip1 mut served as controls. (Fig. 
4A). SA levels were significantly elevated in Zip1-treated samples compared to both mocktreated samples and the Zip1 $1_{\text {mut }}$ controls, demonstrating a specific accumulation of SA upon treatment with the Zip1 peptide (Fig. 4A).

Our observation that Zip1 elicits SA accumulation suggests that its perception also causes a much larger transcriptional response beyond the induction of $P R$-genes. We therefore performed whole transcriptome analyses using Illumina-RNA-Sequencing (RNAseq), which revealed 2713 differentially regulated maize genes in response to SA, compared to mocktreated leaf samples at 24 hours after treatment. Zip1 treatment resulted in 2980 differentially regulated genes compared to mock treatment (Table S1). Remarkably, only 56 genes showed significant differential expression between SA and Zip1 treatments. A comparison of Zip1/SA induced genes to the mock-treated control revealed that $21 \%$ of the differentially regulated genes are exclusively induced in either SA or Zip1 treated samples, respectively (Fig. 4B). Eighty-nine percent of the top-300 upregulated genes are shared between SA and Zip1 treatment. Similarly, $86 \%$ of the top-50 downregulated genes are shared amongst both samples. This surprising and extensive overlap in transcriptional responses induced by both signals demonstrates that Zip1 strongly promotes SA-triggered defence responses in maize. The observed induction of SA accumulation in response to Zip1 (Fig. 4A) is reflected by the transcriptional induction of predicted maize SA biosynthesis key genes ZmPAL1 (Phenylalanine Ammonia-Lyase1) and ZmPAL4 (Table S2). GO enrichment analyses of biological processes (BP) further substantiate these findings. Nitrogen metabolic processes and DNA synthesis, as well as genes associated with translation are downregulated by both Zip1 and SA. BPs upregulated by Zip1 and SA treatment include mainly defence responses ranging from response to fungi, bacteria and biotic stress to cell wall organization and biogenesis (Fig. 4C).

As a confirmation of the RNAseq results, PR-genes analysed by qPCR for the characterization of Zip1 responses (Fig. 1B) were also predictably up-regulated in both Zip1 and SA treatments (Table S2). Most of the SA and Zip1-upregulated transcripts encode for defence genes including 
catalytic and stress protective enzymes like chitinases, $\beta-1,3-g l u c a n a s e s$, peroxidases, heatshock proteins, glutathione S-transferases (GSTs) and other well-known SA markers. In addition, several uncharacterized maize WRKY transcription factors are induced upon SA and Zip1 treatment, whereas two of these are uniquely up-regulated in Zip1-treated samples (Table S2). In summary, RNAseq analyses reveal numerous responses downstream of Zip1, an apoplastic signal that specifically induces SA-dependent gene expression in maize (Fig. 4 and S7). Moreover, Zip1 may also influence ZmPep-mediated defence responses as the ZmPep receptor, ZmPEPR1 as well as its potential co-receptor ZmBAK1 are upregulated by Zip1 (Fig. S7) ${ }^{37,38}$.

Given that Zip1 activates SA signalling, we hypothesized that Zip1 may trigger overall maize immune responses similar to SA. We therefore pre-treated maize leaves with SA, Zip1, Zip1 mut or mock before subsequent infection with the fungal necrotroph Botrytis cinerea. Necrotic lesions caused by $B$. cinerea were quantified 4 days after infection to determine the impact of Zip1 as well as SA. SA pre-treated leaves showed about 2.5 -fold increase in necrotic lesion area compared to buffer treated control plants (Fig. 5A). Strikingly, the lesion size of Zip1 treated leaves displayed a 4-fold increase compared to mock treatments, while Zip1 $1_{\text {mut }}$ challenged leaves did not show an elevated susceptibility to $B$. cinerea compared to mock controls (Fig. 5A). Complementary to an increased susceptibility towards a necrotroph, the proposed function of Zip1 suggests a negative impact on biotrophic interactions. This was tested via the recently established "Trojan horse" (TH) strategy, which deploys recombinant $U$. maydis strains to deliver bioactive plant peptides into the maize apoplast (van der Linde et al., revised). Strikingly, infection of a $U$. maydis mutant expressing secreted Zip1 during infection resulted in a strongly reduced virulence (Fig 5B), as well as elevated expression of $P R$-genes (Fig 5C). Together, these experiments demonstrate that Zip1 activity closely mirrors SA signalling and predictably promotes disease caused by necrotrophic and biotrophic fungi ${ }^{10}$. 


\section{Discussion}

236 The activation and re-localization of plant proteases during pathogen attack has been observed 237 in a wide variety of plant species ${ }^{6}$. We previously demonstrated that apoplastic PLCPs can activate SA-mediated defence signalling in maize and inhibition of these proteases is a crucial step in suppressing immunity and enabling successful infection by biotrophic fungi ${ }^{23,24}$. Within this framework, we proposed two mechanistic scenarios for PLCP action, (a) proteolytic shedding of extracellular receptor domains ${ }^{39,40}$, and (b) activation of peptide hormone signalling by proteolysis of a precursor peptide ${ }^{5,41,42}$. Our current work provides strong support for the second hypothesis, namely SA-induced PLCPs activate the production of peptide signals that further amplify SA production and SA-associated defence responses. Specifically, we identified Zip1 as a signalling peptide mediating SA-dependent immunity, which is released by SA activated PLCPs and, in turn, results in a positive feedback loop amplifying SA-related defence responses in maize (Fig. 5B). It was previously shown that exogenously applied SA mediates activation of five apoplastic PLCPs. Upon activation PLCPs promote SA-dependent PR-gene expression when infiltrated into naïve plants ${ }^{23}$. Through PROZIP1 cleavage studies, we demonstrate that the mixture of apoplastic PLCPs, as well as active form of two recombinant apoplastic PLCPs, namely CP1 and CP2, cleave the propeptide PROZIP1. This event releases bioactive peptides that act as signals to induce SA-associated defence responses which include the reciprocal activation of PLCP activity similar to action of free SA. Using mass spectrometry 254 we were able to detect the 17aa Zip1 peptide as biologically active component in apoplastic 255 fluids of maize leaves. Biological assays however indicated that also a 20aa Zip1 version with 256 three additional $\mathrm{N}$-terminal residues has similar biological activity. This indicates variability of the 257 Zip1 N-terminus, which might result from secondary cleavage by yet unknown proteases. The 258 role of Zip1 in signal amplification explains why apoplastic maize PLCPs are important effector targets. The previously characterized $U$. maydis effector Pit2, as well as the endogenous JAinduced protein ZmCC9 are secreted to the apoplast to establish biotrophic interactions by 
261 blocking apoplastic PLCPs. Thereby the immune response amplifier Zip1 cannot be released 262 from the PROZIP1 precursor protein. In turn, reduced levels of Zip1 impair further SA production 263 and ultimately SA-mediated immunity is dampened ${ }^{24}$. Future work will aim to specify the exact 264 cleavage process of PROZIP1 by generating several cleavage site mutants and test them in 265 cleavage assays with maize PLCPs. Recently, substrate specificity for two PLCPs of Nicotiana 266 benthamiana (NbCysP6, NbCysP7) was analysed in detail ${ }^{43}$. For NbCysP6, which is closely 267 related to maize CP1 a substrate preference for P2-position was identified (L,V or $\mathrm{F})$. While this 268 is in agreement with the predicted N-terminal cleavage site of Zip1, the C-terminal cleavage site 269 (R104 of PROZIP1) is rather unexpected. One possible explanation for this would be that 270 additional plant proteases (e.g. subtilases), which might be activated by the PLCPs, are also 271 involved in the release of the Zip1 peptide.

272 How precisely Zip1 promotes SA production remains unknown. In the context of pathway 273 regulation, the majority of pathogen-induced SA is synthesized from isochorismate produced by 274 isochorismate synthase (ICS) and partially from cinnamate produced by phenylalanine lyase $275(\mathrm{PAL}){ }^{44}$. In line with this is a previous finding that $U$. maydis secretes a chorismate mutase 276 (Cmu1) into maize cells where it re-channels metabolism to lower the substrate availability for 277 SA synthesis ${ }^{45}$. Activity of Cmu1 might also be the reason for a non-complete loss-of-virulence 278 of Zip1-expressing U. maydis strain. A possible scenario would be that Cmu1 activity 279 counteracts the Zip1-induced SA-accumulation allowing a residual level of infection.

280 RNAseq analyses revealed the transcriptional induction of two genes encoding for ZmPAL1 and 281 ZmPAL4 by Zip1 (Table S1,S2). Additionally, ZmPEPR1, a component of peptide induced 282 immune amplification and its potential co-receptor $Z m B A K 1$ are upregulated by SA as well as 283 Zip1 (Table S1, S2) ${ }^{37}$. In contrast to Pep/PEPR signal amplification, Zip1 not only promotes 284 strong SA signalling but downregulates the expression of an essential enzyme involved in maize 285 JA biosynthesis, namely lipoxygenase 8/tassel seed 1 (Table S1) ${ }^{46}$. In the context of candidate 286 biochemical defences, a terpene synthase homolog, ZmTPS21, is exclusively induced by Zip1 
287 (Table S1). Related terpene synthases in maize, such as ZmTps6/11 are $\beta$-macrocarpene synthases predictably responsible for the production of antifungal phytoalexins, termed zealexins ${ }^{47}$. Silencing of $Z m T p s 6 / 11$ promotes increased susceptibility towards $U$. maydis supporting a role in biochemical immunity ${ }^{48}$. Additionally, two WRKY transcription factors are induced by Zip1 that might be involved in immune signalling (Table S2).

292 Collectively, we have identified a peptide, termed Zip1, which activates salicylic acid mediated defenses. Given that SA-dependent immune signalling is a conserved mechanism in plants, it is surprising that Zip1 has little or no sequence homologs in other plant species.

We speculate that a widely conserved Zip1 sequence in plants would create an accessible evolutionary target for necrotrophic pathogen effectors and manipulation. Importance of Zip1 for induction of pathogen induced immunity might also be reflected by an additional copy of the PROZIP1 gene on maize chromosome 8 (GRMZM2G140153; PROZIP2), carrying a single conservative amino acid difference in the coding region (PROZIP1 Ala100 to Val; Fig S7). Presence of an expressed backup copy on a different chromosome further supports the functional importance of Zip1 (Fig S7). Given this potential "Achilles heel" be used by necrotrophs to promote susceptibility, Zip1 function rather than sequence may be conserved as it has been shown for tomato systemin and hydroxyproline-rich glycopeptide systemins (HypSys) ${ }^{49-51}$. Sytemin and HypSys do not share sequence similarities but are both involved in JA-dependent signalling against herbivorous and pathogen attack including systemic synthesis of protease inhibitors and defensins ${ }^{50,52}$. Similar to the systemin-related peptides, additional 307 research is required to determine how Zip1 is perceived by plant cells and to elucidate key signalling nodes responsible for Zip1-induced SA production. Collectively, our current study fills an important conceptual and mechanistic gap in the understanding of how plant apoplastic proteases promote SA signalling. Based on these findings, we are proposing a model on Zip1mediated defenes signalling in maize (Fig 5D). In this scenario, an initial SA burst leads to the

312 activation of apoplastic PLCPs, whichresults in processing of the precursor PROZIP1 to release 
313 the Zip1 peptide signal acting as an amplifier of defense responses to further promote SA 314 production. With predictably important roles in balancing effective defences against biotrophs 315 with susceptibility to necrotrophs, endogenous peptide signals that amplify SA-responses are 316 likely to await discovery in numerous plants. The current discovery of Zip1 provides an 317 important conceptual example of the previously missing intermediate signal that links the 318 activation of apoplastic PLCPs to amplified SA signalling and ultimately inducible plant immune 319 responses.

\section{$321 \quad$ Materials and Methods}

\section{Plant treatments}

323 For all experiments maize plants (Zea mays cv Early Golden Bantam) were grown in a walk-in 324 Phytochamber at $28^{\circ} \mathrm{C}$ during a light period of $12 \mathrm{~h}$ with one hour of twilight, and $22^{\circ} \mathrm{C}$ during a 325 dark period of $11 \mathrm{~h}$. For each experiment the $2^{\text {nd }}$ and $4^{\text {th }}$ leaf of $10-14$ days old plants were taken 326 for analyses. Plants were syringe infiltrated with $2 \mathrm{mM}$ salicylic acid or mock $(0.1 \%$ of EtOH in $327 \mathrm{H}_{2} \mathrm{O}$ ). Treated leaf areas were excised $24 \mathrm{~h}$ after treatment and apoplastic fluid was collected 328 from leaves through centrifugation. Protein content was adjusted to $4.5 \mathrm{mg} \mathrm{ml}^{-1}$. For subsequent 329 qRT-PCR analyses, SA treated leaf tissue was collected 3-4 cm distant from site of infiltration. 330 Individual peptides were synthesized by Genscript Biotech Incorporation (Nanjing, China) and 331 dissolved in $\mathrm{H}_{2} \mathrm{O}$. Leaf infiltration treatments were performed using a blunt needless syringe. 332 Briefly the $2^{\text {nd }}$ and $4^{\text {th }}$ leaves of $1-2$ week old plants were infiltrated with either mock solution or 5 $333 \mu \mathrm{M}$ peptide solutions at the base of the leaf and harvested $24 \mathrm{~h}$ later. Twelve leaves were pooled per sample and treatment for each of five independent biological replicates.

\section{Identification of Z. mays immune signaling peptides and protein precursors}

337 To identify maize peptide signal candidates by mass spectrometry, leaf apolastic fluid of SA or mock treated plants was extracted. Peptide fractions were enriched by filtration using a $10 \mathrm{kDa}$ 
Amicon Centrifugation Filter (EMD Millipore, Darmstadt, Germany) and the application of $5 \mathrm{ml}$ samples of apoplastic fluid, corresponding to $4.5 \mathrm{mg}$ total protein. The $<10 \mathrm{kDa}$ apoplastic fraction was adjusted to a final concentration of $0.5 \%$ formic acid (FA) and $5 \%$ acetonitrile 342 (ACN). The acidified peptide solution was passed in $150 \mu \mathrm{L}$ steps over pre-equilibrated C18 343 spin columns. Next, the columns were washed with $4 \times 0.5 \%$ FA, $5 \%$ ACN to remove excess 344 salts. Finally the bound peptides were eluted with $2 \times 50 \mu \mathrm{L} 0.1 \%$ FA, $70 \%$ ACN and 345 concentrated until $<5 \mu \mathrm{L}$ liquid remained. The resulting volume was then adjusted to $20 \mu \mathrm{L}$ by 346 adding $0.1 \%$ FA. LC-MS/MS-experiments were performed on a Thermo LTQ Velos mass spectrometer coupled to a Proxeon EASY-nLC. Peptides were separated on a single reverse phase C18 column (inner diameter $75 \mathrm{~mm}$, packed with 12-cm ReproSil- Pur C18-AQ [3 $\mu \mathrm{m}]$ ) using an acetonitrile gradient (120 min 5 to $80 \% ; 20$ min $80 \%$ ), at a flow rate of $300 \mathrm{nl} \mathrm{min}^{-1}$. Peptides were fragmented by collision-induced decay in a data-dependent fashion, fragmenting the 20 most intense multiply charged precursors in each MS scan. MS ${ }^{2}$ spectra data were searched using the MASCOT algorithm (version 2.3.02) first against a database of known contaminants (as incorporated in MASCOT) followed by searching against the maize sequences from the database ZmB73_5b_FGS_translations_20110205.fasta

355 (www.maizesequence.org/index.html).

\section{Expression and purification of PROZIP1/PROZIP1mut ${ }^{\mathrm{CS}}$ /PROZIP1mut ${ }^{\mathrm{cS} 2}$}

358 For heterologous protein expression followed by purification, PROZIP1 was amplified from Early 359 Golden Bantam cDNA using oligonucleotides PROZIP1-f and PROZIP1-r (see Table S3). 360 Putative cleavage sites were substituted to alanine in silico and resulting gene was synthesized 361 by Genscript Biotech Incorporation (Nanjing, China). The PROZIP1/ 362 PROZIP1mut ${ }^{\mathrm{CS}} / \mathrm{PROZIP1}$ mut ${ }^{\mathrm{CS} 2}$ proteins were purified via glutathione resin and cleavage of 363 GST-tag was performed as described previously ${ }^{24}$. Further purification of 364 PROZIP1/PROZIP1mut ${ }^{\mathrm{CS}}$ was achieved by gel filtration chromatography on an ÄKTA sytem 
(GE Healthcare Life Science, Buckinghamshire Great Britain) using a Superdex 75 16/600 column equilibrated with storage buffer containing $300 \mathrm{mM} \mathrm{NaCl}, 100 \mathrm{mM}$ Tris-HCl, pH 8.5.

\section{Protease activity assays, ABPP and protease cleavage assays}

To analyze the activity of different cysteine protease, apoplastic fluid from SA treated plants was extracted as described previously ${ }^{23}$ in the presence or absence of E-64 (Sigma-Aldrich, St. Louis, MO, USA) using $10 \mu \mathrm{M}$ of the following substrates: Z-Phe-Arg-7-amido-4-methylcoumarin (AMC), Z-Arg-Arg-AMC, Boc-Gln-Ala-Arg-AMC, N-Succinyl-Leu-Leu-Val-Tyr-AMC (SigmaAldrich, St. Louis, MO, USA). For activity based protein profiling, leaf tissue treated with either Zip1 or SA was used for total protein extraction in $\mathrm{H}_{2} \mathrm{O}+1 \mathrm{mM}$ DTT. Protein concentration was adjusted to $0.2 \mathrm{mg} \mathrm{ml}^{-1}$ with $15 \mathrm{mM}$ sodium phosphate buffer, $\mathrm{pH} 6.0,0.2 \mathrm{mM}$ DTT and preincubated with $5 \mu \mathrm{M} \mathrm{E}-64$ or control buffer in a total volume of $200 \mu \mathrm{L}$ for $30 \mathrm{~min}$ at room temperature prior to the addition of $0.2 \mu \mathrm{L}$ of $2 \mathrm{mM}$ DCG-04. After incubation for $3 \mathrm{~h}$ at room temperature, proteins were precipitated with acetone and resolved in $2 x$ Laemmli loading buffer. $15 \mu \mathrm{L}$ of dissolved proteins were subjected to SDS-PAGE. Immunoblotting and detection of DCG-04 labeled proteins was performed as described in previously ${ }^{23}$. Biotinylated proteins were detected by strep-HRP (1:3000) (Sigma-Aldrich, St. Louis, MO, USA).

For the in vitro cleavage assays $5 \mu \mathrm{M}$ of purified PROZIP1/PROZIP1mutCS/PROZIP1mut ${ }^{\mathrm{CS} 2}$ protein was either incubated with apoplastic fluid from SA treated maize leaves containing active PLCPs, or with apoplastic fluid from $N$. benthamiana leaves transiently expressing individual proteases $\mathrm{CP} 1, \mathrm{CP} 2, \mathrm{XCP} 2$ or CatB according to ${ }^{24}$.

\section{Data availability}

Mass spectrometry and RNA sequencing data availability. Raw read sequences have been deposited in the Sequence Read Archive (SRA) under the BioProject ID PRJNA379074 
390 (https://www.ncbi.nlm.nih.gov/bioproject/PRJNA379074). Data can be accessed under the 391 following collaborator link :

392 Study SRP101910: RNA-seq of Zea mays treated with SA, Zip1 or mock: $393 \quad \underline{\mathrm{ftp}: / / \mathrm{ftp}-}$

394 trace.ncbi.nlm.nih.gov/sra/review/SRP101910 20170711152605 b1659515b9d1a59ebbc790e

$395 \underline{01084 a 8 f 0}$

397 The detailed experimental protocols and methods applied in this study can be found in 398 the Supplementary information.

400 Acknowledgements

401 This work is funded by the German Research Foundation (DFG) via grant DO 1421/5-1 (GD). 402 Mass spectrometry work was financially supported by an ERC starting grant (M.K., grant No. 403 258413) and the Deutsche Forschungsgemeinschaft (M.K., grant no. INST 20876/127-1 404 FUGG). Research in the Zipfel laboratory is supported by the Gatsby Charitable Foundation. 405 We are very grateful to Regine Kahmann for helpful discussions and the Max-Planck-Institute 406 for Terrestrial Microbiology, Marburg, Germany, for continuous support and access to laboratory 407 facilities. We are also very thankful to Alexandra Matei for meaningful discussions and for 408 critical reading the manuscript. We thank Renier van der Hoorn (Oxford University) for 409 generously providing us with ABPP probes. 410.

\section{Authors contribution}

412 S.Z, K.L., and G.D. designed the experiments and analysed the data. S.Z., K.L. and B.A. 413 performed the functional analysis Zip1 / PROZIP1; N.H. and CZ designed and analysed ROS 414 and MAPK assays; Y.D., A.H., and E.S. designed, performed and analysed salicylic acid 
415

416 417 all authors.

418

419 Competing financial interests.

420 No financial interests are declared.

421

422

423

424

425

426

427

428

429

430

431

432

433

434

435

436

437

438

439

440

441

442

443

444

445

446

447

448

449

450

451

452

453

454

\section{Materials \& Correspondence} koeln.de)

\section{References} (2009). Biol 20, 64-68, (2014).

measurements; U.L. analysed the transcriptome data; F.K., T.C. and M.K. performed MS experiments and MS related data analysis; S.Z. and G.D. wrote the manuscript with input from

Correspondence and material requests should be sent to: G. Doehlemann (g.doehlemann@uni-

1 van der Hoorn, R. A. L. Plant proteases: from phenotypes to molecular mechanisms. Annu Rev Plant Biol 59, 191-223, (2008).

2 Xia, Y. et al. An extracellular aspartic protease functions in Arabidopsis disease resistance signaling. EMBO J 23, 980-988, (2004).

3 Tian, M., Huitema, E., da Cunha, L., Torto-Alalibo, T. \& Kamoun, S. A Kazal-like Extracellular Serine Protease Inhibitor from Phytophthora infestans Targets the Tomato Pathogenesis-related Protease P69B. J. .Biol. Chem. 279, 26370-26377, (2004).

4 Tornero, P., Conejero, V. \& Vera, P. Primary structure and expression of a pathogeninduced protease (PR-P69) in tomato plants: similarity of functional domains to subtilisinlike endoproteases. Proc Natl Acad Sci USA 93, 6332-6337, (1996).

5 Stegmann, M. et al. The receptor kinase FER is a RALF-regulated scaffold controlling plant immune signaling. Science 355, 287-289, (2017).

6 Misas-Villamil, J. C., van der Hoorn, R. A. L. \& Doehlemann, G. Papain-like cysteine proteases as hubs in plant immunity. New Phytologist, n/a-n/a, (2016).

7 Rooney, H. C. et al. Cladosporium Avr2 inhibits tomato Rcr3 protease required for Cf-2dependent disease resistance. Science 308, 1783-1786, (2005).

8 Song, J. et al. Apoplastic effectors secreted by two unrelated eukaryotic plant pathogens target the tomato defense protease Rcr3. Proc Natl Acad Sci U S A 106, 1654-1659,

9 Lozano-Torres, J. L. et al. Dual disease resistance mediated by the immune receptor Cf2 in tomato requires a common virulence target of a fungus and a nematode. Proc Natl Acad Sci U S A 109, 10119-10124, (2012).

10 Glazebrook, J. Contrasting mechanisms of defense against biotrophic and necrotrophic pathogens. Annual review of phytopathology 43, 205-227, (2005).

11 Pieterse, C. M., Leon-Reyes, A., Van der Ent, S. \& Van Wees, S. C. Networking by small-molecule hormones in plant immunity. Nature chemical biology 5, 308-316, (2009).

12 Yan, S. \& Dong, X. Perception of the plant immune signal salicylic acid. Curr Opin Plant 
491

492

493

494

495

496

497

498

499

500

501

502

503

504

13 Doherty, H. M., Selvendran, R. R. \& Bowles, D. J. The wound response of tomato plants can be inhibited by aspirin and related hydroxy-benzoic acids. Phys and Mol Plant Pathol 33, 377-384, (1988).

14 Pefia-Cortes, H., Albrecht, T., Prat, S., Weiler, E. W. \& Willmitzer, L. Aspirin prevents wound-induced gene expression in tomato leaves by blocking jasmonic acid biosynthesis. Planta 191, 123 128, (1993).

15 Thomma, B. P. et al. Separate jasmonate-dependent and salicylate-dependent defenseresponse pathways in Arabidopsis are essential for resistance to distinct microbial pathogens. Proc Natl Acad Sci USA 95, 15107-15111, (1998).

16 Huffaker, A. et al. Plant elicitor peptides are conserved signals regulating direct and indirect antiherbivore defense. Proc Natl Acad Sci U S A 110, 5707-5712, (2013).

17 Huffaker, A., Dafoe, N. J. \& Schmelz, E. A. ZmPep1, an ortholog of Arabidopsis elicitor petide 1 , regulates maize innate immunity and enhances disease resistance. Plant physiology 155, 1325-1338, (2011).

18 Huffaker, A., Pearce, G. \& Ryan, C. A. An endogenous peptide signal in Arabidopsis activates components of the innate immune response. Proc Natl Acad Sci USA 103, 10098-10103, (2006).

19 Brefort, T. et al. Ustilago maydis as a pathogen. Annual review of phytopathology 47, 423-445, (2009).

20 Doehlemann, G. et al. Reprogramming a maize plant: transcriptional and metabolic changes induced by the fungal biotroph Ustilago maydis. Plant J 56, 181-195, (2008).

21 Hof, A., Zechmann, B., Schwammbach, D., Huckelhoven, R. \& Doehlemann, G. Alternative cell death mechanisms determine epidermal resistance in incompatible barley-ustilago interactions. Molecular plant-microbe interactions : MPMI 27, 403-414, (2014).

22 Doehlemann, G. et al. Pep1, a secreted effector protein of Ustilago maydis, is required for successful invasion of plant cells. PLoS pathogens 5, e1000290, (2009).

23 van der Linde, K. et al. A maize cystatin suppresses host immunity by inhibiting apoplastic cysteine proteases. The Plant cell 24, 1285-1300, (2012).

24 Mueller, A. N., Ziemann, S., Treitschke, S., Assmann, D. \& Doehlemann, G. Compatibility in the Ustilago maydis-maize interaction requires inhibition of host cysteine proteases by the fungal effector Pit2. PLoS pathogens 9, (2013).

25 Dolezal, A. L. et al. Aspergillus flavus infection induces transcriptional and physical changes in developing maize kernels. Front Microbiol 5, 384, (2014).

26 Ray, S. et al. Turnabout Is Fair Play: Herbivory-Induced Plant Chitinases Excreted in Fall Armyworm Frass Suppress Herbivore Defenses in Maize. Plant physiology 171, 694706, (2016).

27 Barrett, A. J., Kembhavi, A. A. \& Hanada, K. E-64 [L-trans-epoxysuccinyl-leucylamido(4-guanidino)butane] and related epoxides as inhibitors of cysteine proteinases. Acta Biol Med Ger 40, 1513-1517, (1981).

28 Cravatt, B. F., Wright, A. T. \& Kozarich, J. W. Activity-based protein profiling: from enzyme chemistry to proteomic chemistry. Annu Rev Biochem 77, 383-414, (2008).

29 Greenbaum, D., Medzihradszky, K. F., Burlingame, A. \& Bogyo, M. Epoxide electrophiles as activity-dependent cysteine protease profiling and discovery tools. Chem Biol 7, 569-581, (2000).

30 Paireder, M. et al. The death enzyme CP14 is a unique papain-like cysteine proteinase with a pronounced S2 subsite selectivity. Arch Biochem Biophys 603, 110-117, (2016).

31 Choe, Y. et al. Substrate profiling of cysteine proteases using a combinatorial peptide library identifies functionally unique specificities. The Journal of biological chemistry 281, 12824-12832, (2006). 
32 Zipfel, C. et al. Perception of the bacterial PAMP EF-Tu by the receptor EFR restricts Agrobacterium-mediated transformation. Cell 125, 749-760, (2006).

33 Zipfel, C. et al. Bacterial disease resistance in Arabidopsis through flagellin perception. Nature 428, 764-767, (2004).

34 Monaghan, J. et al. The calcium-dependent protein kinase CPK28 buffers plant immunity and regulates BIK1 turnover. Cell host \& microbe 16, 605-615, (2014).

35 Miya, A. et al. CERK1, a LysM receptor kinase, is essential for chitin elicitor signaling in Arabidopsis. Proc Natl Acad Sci 104, 19613-19618, (2007).

36 Jabaiah, A. M., Getz, J. A., Witkowski, W. A., Hardy, J. A. \& Daugherty, P. S. Identification of protease exosite-interacting peptides that enhance substrate cleavage kinetics. Biol Chem 393, 933-941, (2012).

37 Lori, M. et al. Evolutionary divergence of the plant elicitor peptides (Peps) and their receptors: interfamily incompatibility of perception but compatibility of downstream signalling. Journal of experimental botany 66, 5315-5325, (2015).

38 Bartels, S. \& Boller, T. Quo vadis, Pep? Plant elicitor peptides at the crossroads of immunity, stress, and development. Journal of experimental botany 66, 5183-5193, (2015).

39 Cirino, G. \& Vergnolle, N. Proteinase-activated receptors (PARs): crossroads between innate immunity and coagulation. Curr Opin Pharmacol 6, 428-434, (2006).

40 Schmidlin, F. \& Bunnett, N. W. Protease-activated receptors: how proteases signal to cells. Current Opinion in Pharmacology 1, 575-582, (2001).

41 Srivastava, R., Liu, J. X., Guo, H., Yin, Y. \& Howell, S. H. Regulation and processing of a plant peptide hormone, AtRALF23, in Arabidopsis. Plant J 59, 930-939, (2009).

42 Srivastava, R., Liu, J. X. \& Howell, S. H. Proteolytic processing of a precursor protein for a growth-promoting peptide by a subtilisin serine protease in Arabidopsis. Plant $J \mathbf{5 6}$, 219-227, (2008).

43 Paireder, M. et al. The papain-like cysteine proteinases NbCysP6 and NbCysP7 are highly processive enzymes with substrate specificities complementary to Nicotiana benthamiana cathepsin B. Biochim Biophys Acta 1865, 444-452, doi:10.1016/j.bbapap.2017.02.007 (2017).

44 Chen, Z., Zheng, Z., Huang, J., Lai, Z. \& Fan, B. Biosynthesis of salicylic acid in plants. Plant signaling \& behavior 4, 493-496, (2009).

45 Djamei, A. et al. Metabolic priming by a secreted fungal effector. Nature 478, 395-398, doi:10.1038/nature10454 (2011).

46 Acosta, I. et al. tasselseed1 Is a Lipoxygenase Affecting Jasmonic Acid Signaling in Sex Determination of Maize. Science 323, 262-265, (2009).

47 Huffaker, A. et al. Novel acidic sesquiterpenoids constitute a dominant class of pathogen-induced phytoalexins in maize. Plant physiology 156, 2082-2097, (2011).

48 van der Linde, K., Kastner, C., Kumlehn, J., Kahmann, R. \& Doehlemann, G. Systemic virus-induced gene silencing allows functional characterization of maize genes during biotrophic interaction with Ustilago maydis. The New phytologist 189, 471-483, (2011).

49 Pearce, G. et al. Isolation and characterization of hydroxyproline-rich glycopeptide signals in black nightshade leaves. Plant physiology 150, 1422-1433, (2009).

50 Pearce, G., Strydom, D., Johnson, S. \& Ryan, C. A. A polypeptide from tomato leaves induces wound-inducible proteinase inhibitor proteins. Science 253, 895-897, (1991).

51 Ryan, C., Pearce, G., Scheer, J. \& Moura, D. Polypeptide Hormones. the Plant Cell Online, 251-264, (2002).

52 Chen, Y. C., Siems, W. F., Pearce, G. \& Ryan, C. A. Six peptide wound signals derived from a single precursor protein in Ipomoea batatas leaves activate the expression of the defense gene sporamin. The Journal of biological chemistry 283, 11469-11476, (2008). 
555

556

557

558

559

560

561

562

\section{Figure Legends}

\section{Fig. 1. Induction of SA-associated PR-gene expression by apoplastic peptide fraction as}

well as by Zip1 [A] qRT-PCR analyses of maize leaves treated with apoplastic peptide fractions from SA-treated leave samples show induction of SA-associated PR-gene expression (PR3, PR4 and PR5; black bars) compared to peptides of mock treated samples (grey bars). CC9 as a control for JA-marker genes is not induced. [B] Maize leaves were treated with $5 \mu$ M Zip1 (dark grey) and $5 \mu \mathrm{M}$ Zip1mut (light grey) as well as with $2 \mathrm{mM} \mathrm{SA}$ (black). Peptide treatment and subsequent qRT-PCR analyses reveals Zip1 to be capable to induce SA-associated PR-gene expression in maize leaves 24 hours after treatment. Charged N-terminal amino acids (red) are essential to maintain biological activity of Zip1 as Zip1mut is not inducing PR-gene expression. Experiments shown in this figure were done in five independent biological replicates with two technical replicates in each measurement; error bars represent SEM; $p$-values were calculated by an unpaired $t$-test. ${ }^{*} P<0.05 ;{ }^{*} P<0.005 ;{ }^{* *} P<0.0005$

\section{Fig. 2. Active PLCPs are required for processing of PROZIP1. [A] Heterologously expressed} PROZIP1 $(5 \mu \mathrm{M})$ was co-incubated with AF of SA-treated maize leaves containing active PLCPs. 0, 5 and 15 min timepoints were analysed using $\alpha-H A$ western blot. Activity of PLCPs was monitored by ABPP using DCG-04, a specific probe for the detection of active PLCPS. PLCPs efficiently process PROZIP1 over time, which can be inhibited by E-64. PROZIP1mutCS with putative cleavage sites mutated is not cleaved anymore. [B] Individual PLCPs were heterologously expressed in $N$. benthamiana via $A$. tumefaciens-mediated transformation. Activity of CP1, CP2, CatB and XCP2 was normalized and examined by ABPP using MV-202 as fluorescent probe. [C] PROZIP1, PROZIP1mut ${ }^{\mathrm{CS}}$ as well as PROZIP1mut ${ }^{\mathrm{CS} 2}$ carrying an Nterminal HA epitope were tested in in vitro cleavage assays with individual proteases. $\alpha-\mathrm{HA}$ 
580 immunoblotting shows that $\mathrm{CP} 2$ and $\mathrm{CP} 1$, but not CatB and XCP2 are responsible for PROZIP1

581 cleavage. PROZIP1 ${ }^{\text {mutcs }}$ with all RR motifs mutated is not processed whereas PROZIP1mut ${ }^{\mathrm{CS} 2}$

582 is cleaved although slightly less than wild type PROZIP1. [D] Alignment of PROZIP1 and 583 PROZIP1 variants that were generated in this study. In PROZIP1mut ${ }^{\mathrm{CS}} / \mathrm{PROZIP1mut}{ }^{\mathrm{CS} 2}$ 584 different sets of putative cleavage sites (red) were substituted by Alanine (blue). Zip1 is 585 highlighted in green.

587 Fig. 3. In vitro released Zip1 is active in vivo. [A] PROZIP (10 $\mu \mathrm{M})$, PROZIP1mut ${ }^{\mathrm{CS}}(10 \mu \mathrm{M})$ 588 and PROZIP1mut ${ }^{\mathrm{CS} 2}(10 \mu \mathrm{M})$ were co-incubated with AF fractions containing active PLCPS 589 monitored by ABPP. Subsequently peptide fractions were separated from protein fractions. 590 Maize leaves were treated with each fraction, respectively. 24 hpi qRT-PCR analyses show a 591 significant induction of PR-gene expression with peptide fractions of PROZIP1 cleavage 592 reactions. This effect can be abolished by blocking PLCPs activity with E-64 prior to PROZIP1 593 incubation. PROZIP1 mut ${ }^{\mathrm{CS}}$ and PROZIP1mut ${ }^{\mathrm{CS} 2}$ peptide fractions do not induce a significant SA594 associated defense gene expression. Protein fractions of all PROZIP cleavage reactions do not 595 induce PR-gene expression. The experiments were done in three independent biological 596 replicates; error bars represent SEM; $P$-values were calculated by an unpaired t-test. ${ }^{*} P<0.05$; $597{ }^{* \star} P<0.005$. [B] Zip1 induces PLCP activity. Maize leaves were treated with $5 \mu \mathrm{M}$ Zip1 and 598 Zip1mut as well as 2 mM SA. 24 hpi PLCP activity was monitored via APBB using DCG-04 599 probe. Zip1 induces the activation of PLCPs same as SA does (left panel). To ascertain if Zip1 600 induces PLCP activation by direct interaction, leaf extract of treated plants was co-incubated 601 with Zip1 before ABPP showing no activation of PLCPs by direct interaction with Zip1 (right 602 panel).

603

604 Fig. 4. Zip1 induced accumulation of SA in maize leaves and RNA-sequencing analyses

605 of Zip1 and SA treated maize leaves. [A] Maize leaves were treated with $5 \mu \mathrm{M}$ Zip1 and 
606

Zip1mut. 24 hpi total free SA was measured in mock, Zip1mut and Zip1 treated samples using LC/MS-MS. Zip1 causes a 20-fold accumulation of SA compared to mock. SA induction induced by Zip1 is statistically significant compared to Zip1mut. [B] To identify additional responses mediated by Zip1 whole transcriptome analyses was performed at $24 \mathrm{~h}$ using RNAseq. The upand downregulated genes in SA and Zip1 (compared to mock control) were compared against each other. For this, we took the strongest differentially regulated genes above/below a logFC threshold of $\sim+$ +- 1.6. $266(89 \%)$ of the 300 strongest upregulated genes in SA are also upregulated in Zip1 and $43(86 \%)$ of the 50 strongest downregulated genes in SA are also downregulated in Zip1. Vice versa, 268 (89\%) of the 300 strongest upregulated genes in Zip1 are also upregulated in SA and 36 (72\%) of the 50 strongest downregulated genes in Zip1 are also downregulated in SA. For all comparisons a significant threshold (adj.P) of $<0.05$ was applied. [C] Differential gene expression of GO-term categories between Zip1/Mock and SA/Mock was calculated with R/DESeq2. With all genes differentially regulated under an FDRadjusted significance cutoff level of 0.05 , parametric analysis of gene set enrichment (PAGE) was applied with agriGO, Zea mays AGPv3.30 and the complete GO list. Gene ontologies important in immune response signalling were manually selected and the corresponding Z-score from the PAGE analysis was visualized in a heatmap. Asterisks (*) denote values with an adj. $P$ $\geq 0.05$.

\section{Fig. 5. Zip1 confers increased susceptibility of maize towards the necrotrophic pathogen} Botrytis cinerea but mitigates infection by the biotrophic fungus Ustilago maydis. $[A]$ Maize leaves were pre-treated with $5 \mu \mathrm{M}$ Zip1mut or Zip1 and $2 \mathrm{mM} \mathrm{SA}$, respectivley. 24 hpi pre-treated leaves were detached and infected with $10 \mu \mathrm{L}$ droplets of $B$. cinerea spore solution containing $1 \times 10^{6}$ spores $\mathrm{mL}^{-1}$. In line with SA measurements Zip1 pre-treatment causes higher susceptibility to $B$. cinerea. [B] Maize seedling were infected with biotrophic Ustilago maydis wildtype strain (SG200) and a U. maydis mutant that expresses secreted Zip1. U. maydis Zip1 
632 expressing strain shows strongly reduced tumor formation at $12 \mathrm{dpi}$ in three independent 633 biological replicates. $\mathrm{n}=$ number of plants infected. $P$-values were calculated by an unpaired $\mathrm{t}$ 634 test. ${ }^{*} P<0.05$. [C] qRT-PCR of $U$. maydis infected maize leaves proves that Zip1 secretion by 635 U.maydis induces the expression of SA-associated PR-genes PR3 and PR5 at 2 dpi. The 636 experiments were done in three independent biological replicates; error bars represent SEM; $P$ 637 values were calculated by an unpaired t-test. ${ }^{*} P<0.05$. [D] Model of Zip1-mediated defense 638 signalling in maize. Upon infection biotrophic pathogens such as $U$. maydis trigger JA639 associated defense responses by so far unknown mechanisms. By that, maize endogenous 640 CC9 as well as the U. maydis effector protein Pit2 are induced to inhibit PLCP activity. Likewise, 641 SA signalling is directly suppressed by Cmu1, an effector protein that suppresses SA synthesis. 642 In contrast, induced SA signalling leads to the activation of PLCPs. Thus, PROZIP1 is 643 processed by $\mathrm{CP} 1$ and CP2 which releases active Zip1. Zip1 signalling induces several SA644 associated downstream signalling events and PLCP activation. Together with Zip1-induced 645 accumulation of SA, the newly discovered peptide Zip1 amplifies SA-associated defense 646 responses. 